\title{
Fiber vibration sensor multiplexing techniques for quasi-distributed sensing
}

\begin{abstract}
A multiplexing technique for fiber vibration sensors is experimentally investigated using Khazani Syed (KS) code in SAC/OCDMA with direct decoding. The system is proposed to implement vibration sensor multiplexing which can eliminate the Multiple Access Interference (MAI) at low cost and complexity. The results show the proposed system having better SNR, less complex, and low cost when compared with complementary decoding, and higher power level when compared with simplified WDM. A frequency range of 0 to $400 \mathrm{~Hz}$ measured shows its suitability for quasi-distributed sensing in bridges, pipelines, transformers, and industrial machine that exhibit low vibrations within this range.
\end{abstract}

Keyword: Multiplexing technique; Quasi-distributed sensing; Vibrations 